\title{
Lebrikizumab in the personalized management of asthma
}

\author{
This article was published in the following Dove Press journal: \\ Biologics:Targets and Therapy \\ 13 September 2012 \\ Number of times this article has been viewed
}

\author{
Neil C Thomson' \\ Manish Patel ${ }^{2}$ \\ Andrew D Smith ${ }^{2}$ \\ 'Institute of Infection, Immunity, \\ and Inflammation, University of \\ Glasgow and Respiratory Medicine, \\ Gartnavel General Hospital, Glasgow, \\ UK; ${ }^{2}$ Department of Respiratory \\ Medicine, Wishaw Hospital, Wishaw, \\ Lanarkshire, UK
}

\begin{abstract}
There is a need for improved therapies for severe asthma. Lebrikizumab, a humanized monoclonal antibody that binds to interleukin (IL)-13, is under development for the treatment of poorly controlled asthma. This article reviews the potential role of IL-13 in the pathogenesis of asthma, the efficacy and safety of lebrikizumab in humans, and progress in patient selection for lebrikizumab therapy. IL-13 is a T-helper (Th2) cell-derived cytokine implicated in inflammatory responses in asthma, including serum immunoglobulin-E synthesis, mucus hypersecretion, and subepithelial fibrosis. Blocking the pro-inflammatory effects of IL-13 with lebrikizumab has the potential to improve asthma control. Published data on the efficacy and safety of lebrikizumab in the treatment of asthma are relatively limited. The late asthmatic response after inhaled allergen challenge is reduced by almost 50\%, following treatment with lebrikizumab. In a Phase II study performed in 219 adults with poorly controlled asthma despite inhaled corticosteroids (MILLY trial), lebrikizumab produced an improvement in prebronchodilator forced expiratory volume in 1 second of $5.5 \%$ compared with placebo at 12 weeks, but had no effects on other efficacy end points. Adverse effects were similar to placebo, except that musculoskeletal side effects occurred slightly more often with lebrikizumab. Stratifying patients into a high Th2 phenotype using serum periostin, which is upregulated in lung epithelial cells by IL-13, may identify individuals responsive to blockade of IL-13. In the MILLY trial, lebrikizumab treatment was associated with greater improvement in lung function in patients with elevated serum periostin levels compared with those with low periostin levels. Two large Phase III randomized controlled trials in patients with uncontrolled asthma are underway to establish the safety and efficacy of lebrikizumab when administered over a 52-week period. These studies will also help to determine whether identifying patients with a Th2 high inflammatory phenotype using serum periostin allows a personalized approach to the treatment of asthma.
\end{abstract}

Keywords: asthma, periostin, interleukin-13, phenotypes, exhaled nitric oxide, lebrikizumab

\section{Introduction}

Asthma is a chronic inflammatory condition of the airways characterized by airway hyperresponsiveness, inflammatory infiltrates in the bronchial wall including eosinophils, and elevated serum immunoglobulin-E (IgE) levels in allergic patients. T-helper type 2 (Th2) lymphocytes are thought to play a key role in the initiation and perpetuation of this airway inflammation in asthma (Figure 1). ${ }^{1-3}$ For many years, asthma was considered a single disease. It is now becoming apparent that there is wide heterogeneity in the asthma phenotype, which may well explain the differential responses to established therapies such as inhaled corticosteroids and long-acting beta ${ }_{2}$ agonists (LABA). ${ }^{4-6}$ Even with the introduction of new biological agents, there are still a large
Correspondence: Neil C Thomson Respiratory Medicine, Gartnavel General Hospital and University of Glasgow, Glasgow GI 2 OYN, UK

Tel +44 |4| 2 II 0095

Fax +44 I4I 21 I 3464

Email neil.thomson@glasgow.ac.uk 


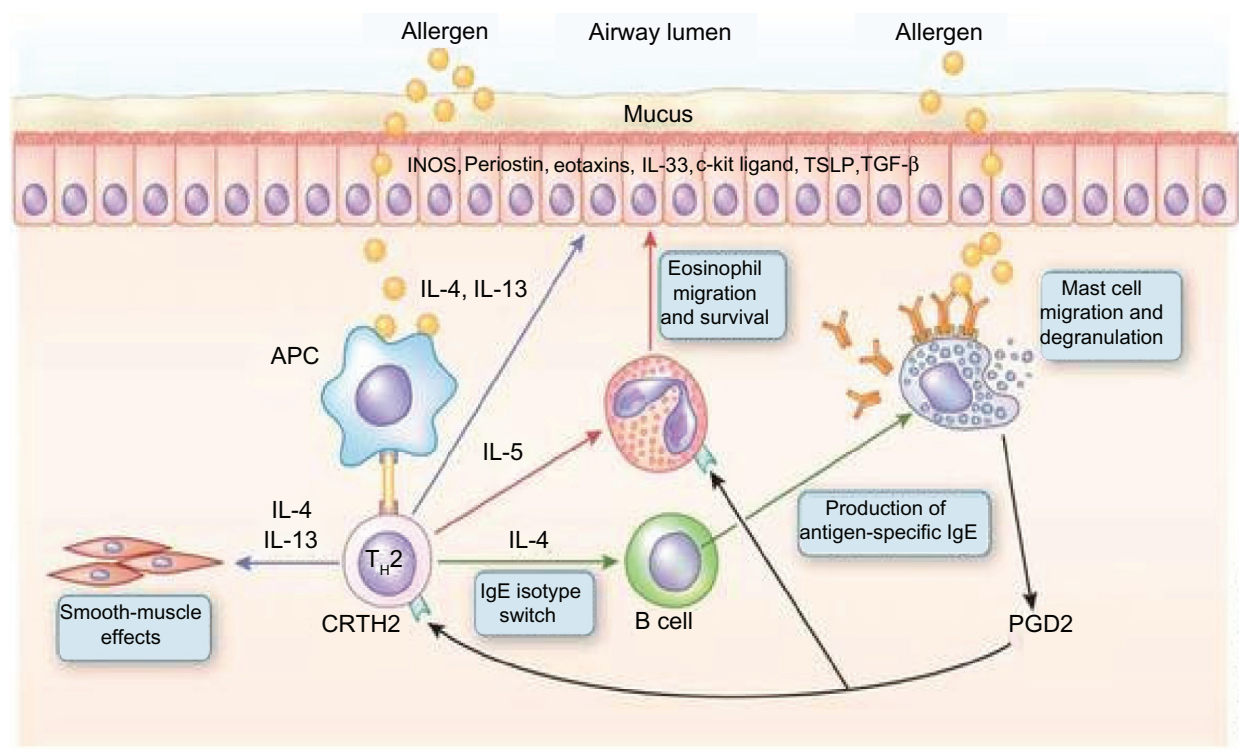

Figure I T helper-2 (Th-2) cells in asthma pathogenesis. Inhaled allergens are thought to be processed by two mechanisms in asthmatic airways.

Notes: Allergens either: (I) activate mast cells through cross-linking with IgE on their cell surfaces through the high-affinity type I IgE receptor (FcER I) to release mediators that induce bronchoconstriction, such as histamine, cysteinyl leukotrienes, and prostaglandin $\mathrm{D}_{2}\left(\mathrm{PGD}_{2}\right)$ or $(2)$ are processed by dendritic cells, which are induced to secrete the CC chemokine ligand (CCL) 17 and CCL22 by thymic stromal lymphopoietin (TSLP). Dendritic cells then attract and activate Th2 cells by the binding of CCLI7 and CCL22 with CC chemokine receptor 4 (CCR4) on the Th2 cell surface. IL-33 is produced by airway epithelial cells and activates dendritic cells and Th2 by inducing the release of tumor necrosis factor-alpha from mast cells. Th2 secretes cytokines, including IL-4 and IL-I3, which switch B cells to produce IgE, IL-5, which promotes the development and survival of eosinophils, and IL-9, which activates mast cells. Once IL-I 3 is produced, it can increase the survival and migration of eosinophils, and it promotes activation of macrophages to create an M2, or an allergic cell phenotype. Airway epithelial cells are stimulated, and through mediators such as periostin and transforming growth factor $\beta 1$ (TGF- $\beta$ I), they can increase airway inflammation and lead to the increased permeability of airway epithelial cells and mucous hypersecretion. IL-I 3 also has direct effects on airway smooth muscle, leading to increased contraction to agonists such as acetylcholine and decreased relaxation with beta-agonists. Adapted by permission from Macmillan Publishers Ltd: Nature Medicine, Wenzel SE. Asthma phenotypes: the evolution from clinical to molecular approaches. Nat Med. 20I2;18(5):716-725, copyright 20I2.39

Abbreviations: APC, antigen-presenting cells; IgE, immunoglobulin E; IL-, interleukin; iNOS, inducible nitric oxide synthase.

number of patients whose asthma remains uncontrolled. For example, up to $40 \%$ of patients with severe allergic asthma do not gain adequate disease control despite the addition of omalizumab, a monoclonal antibody that binds IgE. ${ }^{78}$ Hence, there is still a need for new agents to treat asthma, and an even greater need for agents that are tailored to the individual phenotype of asthmatic patients. ${ }^{9} 10$

One potential target for these novel types of individualized or personalized asthma therapies is interleukin (IL)-13. IL-13 shares structural similarities with IL-4, and it has been clearly demonstrated that it is important in the effector pathways critical in the inflammatory response in murine models of allergic asthma (Figure 1). ${ }^{11,12}$ IL-13 is involved in the regulation of $\operatorname{IgE}$ synthesis, mucus hypersecretion, subepithelial fibrosis, and eosinophil infiltration of asthmatic bronchi. ${ }^{13}$ There are several human studies that demonstrate a link between IL-13 gene polymorphisms and atopy and asthma. ${ }^{14,15}$ It is clear that IL-13 production is increased in human asthma and inhibited by inhaled corticosteroids. ${ }^{16}$ In some patients with more severe asthma, IL-13 levels in the sputum and bronchial biopsy samples remain elevated despite the use of systemic and inhaled corticosteroids. ${ }^{16}$ Hence, IL-13 is considered one of the potential inflammatory mediators involved in corticosteroid resistance. ${ }^{17} \mathrm{IL}-13$ acts on many of the cells in the asthmatic airways, including the airway epithelium. IL-13 induces airway epithelial cells to secrete several matricellular proteins - one of which is periostin. Periostin stimulates epithelial cells and fibroblasts to increase fibrosis and decreases elasticity in the bronchial epithelium, and is thought to be important in airway remodeling in asthma. ${ }^{18-20}$

Asthma is a heterogeneous disease and has historically been regarded as an inflammatory disease mediated by Th2 cells. This may be true for allergic asthma; however, there is a large proportion of patients whose airway inflammation is driven by a noneosinophilic pathway. ${ }^{21-23}$ Even within the atopic phenotype, there is heterogeneity. A "high"type Th2 phenotype has been described by Woodruff and colleagues, ${ }^{24}$ who classified asthmatic individuals based on their expression of IL-13-inducible genes. The high-Th2 phenotype has been defined as an $\operatorname{IgE}$ level greater than $100 \mathrm{ng}$ per milliliter and more than $0.14 \times 10^{9}$ eosinophils per liter in the peripheral blood. ${ }^{24}$ The high-Th2 phenotype has elevated IL-5 and IL-13 levels in bronchial biopsies and increased airway hyperresponsiveness, airway eosinophilia, and subepithelial fibrosis. Asthmatic patients with the 
high-Th2 phenotype also overexpress epithelial proteins, one of which is periostin. ${ }^{24}$

Given the large number of asthmatic patients who remain uncontrolled on conventional therapy, ${ }^{4-7}$ blockade of Th2-derived cytokines would seem an appropriate target for new biological agents. There is very strong evidence for antagonism of Th2 cytokines (IL-4, IL-5, IL-13), and tumor necrosis factor- $\alpha$ in animal models of asthma. Unfortunately, these findings do not translate consistently in human asthma. ${ }^{9,10}$ This variable response in human asthma will no doubt reflect the heterogeneity of asthma phenotypes. Novel therapies for severe asthma are likely to involve genotypic and/or phenotypic assessment to identify patients who will gain the most from a specific intervention. ${ }^{9,25}$

Lebrikizumab, a humanized monoclonal antibody that binds to IL-13, is under development for the treatment of asthma. The purpose of this article is to review the pharmacology and mode of action of lebrikizumab, the efficacy and safety of lebrikizumab in humans, and the progress in patient profiling and selection for lebrikizumab therapy.

\section{Pharmacology of lebrikizumab}

Lebrikizumab (MILR1444A) is an $\mathrm{IgG}_{4}$ humanized monoclonal antibody that binds to IL-13. The molecule was developed by Genentech (South San Francisco, CA). The stability of lebrikizumab is increased by a single joint mutation on the hinge portion of the molecule. The molecular formula for lebrikizumab is $\mathrm{C}_{6434} \mathrm{H}_{9972} \mathrm{~N}_{1700} \mathrm{O}_{2034} \mathrm{~S}_{50}$. In vitro studies demonstrate the ability of lebrikizumab to inhibit the function of IL-13. ${ }^{26}$ IL-13-induced phosphorylation of signal transducer and activator of transcription- 6 in TF- 1 cells, ${ }^{13}$ assessed by flow cytometry, as well as the proliferation of these cells, ${ }^{27}$ assessed by measuring the incorporation of $\mathrm{H} 3$-thymidine, are inhibited by lebrikizumab. ${ }^{26}$

\section{Efficacy studies}

Published data on the efficacy of lebrikizumab in the treatment of asthma are relatively limited to date. ${ }^{26,28}$ In a randomized controlled trial in 28 patients with allergic asthma, the late asthmatic response after inhaled allergen challenge (primary outcome measure) was reduced by $48 \%$ following 3 months of treatment with lebrikizumab compared with placebo (95\% confidence interval [CI]: $-19 \%-90 \%) .{ }^{28}$ Lebrikizumab had no effect on the early asthmatic response. ${ }^{28}$ Systemic biomarkers considered to indicate Th2 inflammation (serum IgE, Cys-Cys ligand [CCL]13 chemokine, and CCL17 chemokine), decreased by $20 \%$ to $25 \%$ after lebrikizumab treatment. ${ }^{28}$ Participants with peripheral blood eosinophils, serum IgE, or serum periostin levels above the median compared to those below this cutoff were reported to obtain a greater decrease in the late asthmatic response after lebrikizumab, suggesting that systemic biomarkers may help predict the magnitude of clinical response to lebrikizumab treatment.

A Phase II randomized, double-blind, placebo-controlled study of lebrikizumab was performed in 219 adults with asthma that was poorly controlled despite inhaled corticosteroids (mean dose: $580 \mu \mathrm{g}$ per day) (NCT00930163; MILLY trial). ${ }^{26}$ Eighty percent of the participants were also taking LABA therapy. Lebrikizumab was administered subcutaneously at a dose of $250 \mathrm{mg}$ monthly for 24 weeks. The primary efficacy outcome measure was the relative change in prebronchodilator forced expiratory volume in 1 second $\left(\mathrm{FEV}_{1}\right)$ from baseline to 12 weeks. Secondary end points included rate of asthma exacerbations from baseline to 24 weeks and morning prebronchodilator peak exploratory flow, change in Asthma Control Questionnaire (ACQ) score, asthma symptom score, use of recue medication, and measurement of the fraction of exhaled nitric oxide $\left(\mathrm{Fe}_{\mathrm{NO}}\right)$ from baseline to 12 weeks. Lebrikizumab treatment produced an improvement in mean (95\% CI: prebronchodilator $\mathrm{FEV}_{1}$ of $5.5 \%$ [0.8 to 10.2]) compared with placebo $(P=0.02)$ (Figure 2). At 20 weeks, the postbronchodilator $\mathrm{FEV}_{1}$ was slightly higher in the lebrikizumab group (3.4\%) compared with placebo (-1.5\%). Treatment with lebrikizumab had no significant effects on the secondary efficacy end points. Lebrikizumab treatment produced a $19 \%$ mean fall in $\mathrm{Fe}_{\mathrm{NO}}$ at 12 weeks compared with a $10 \%$ increase with placebo $(P<0.001)$.

A preliminary report on a further analysis of this study found that lebrikizumab treatment reduced severe asthma exacerbations over 32 weeks ( 24 weeks during the randomized controlled trial plus the post-last dose 8-week follow-up period) in adults with inadequately controlled asthma. ${ }^{29}$ Severe exacerbations were defined as new or worsened asthma symptoms requiring systemic corticosteroid treatment, overnight hospital admission, or institution of highdose inhaled corticosteroid therapy. In all patients $(n=218)$, the severe exacerbation rate over the 32-week period was reduced by $50 \%$ in the lebrikizumab group $(0.17$ exacerbations) compared to the placebo group ( 0.34 exacerbations, $P=0.03)$ - a relative absolute reduction in severe exacerbation of 0.17 . A trend toward a larger reduction in severe exacerbations rates was found in patients with high periostin levels prior to treatment $(61 \%[-1,85], P=0.06)$.

Prior to randomization, the MILLY trial patients were categorized into high-Th2 or low-Th2 status, based on total 

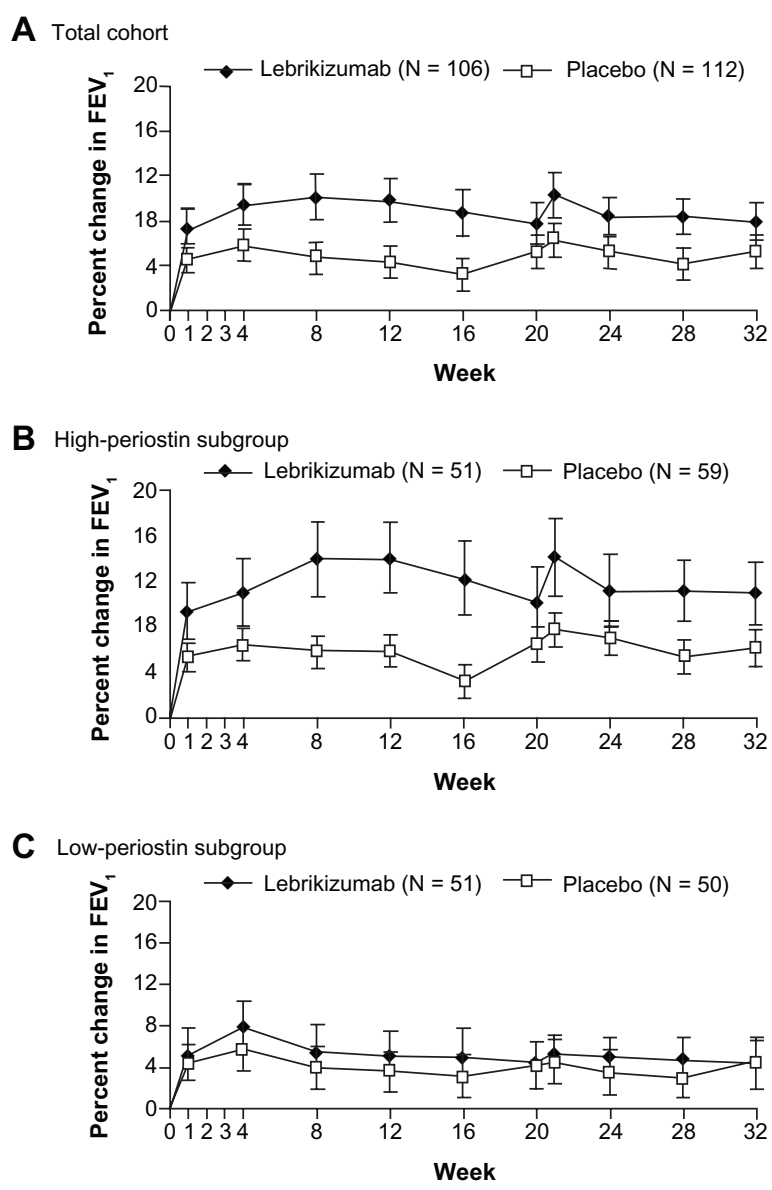

Figure 2 Effect of lebrikizumab treatment on the change in FEV, values in adults with asthma. For all patients at week 12 (A), the increase from baseline in mean ( \pm SE) $\mathrm{FEV}$, was higher in the lebrikizumab group $(9.8 \% \pm 1.9 \%)$ than in the placebo group $(4.3 \% \pm 1.5 \%)(P=0.02)$. In the subgroup of patients with high serum periostin levels at week 12 (B), the increase from baseline in mean ( \pm SE) FEV was higher in the lebrikizumab group $(14.0 \% \pm 3.1 \%)$ than in the placebo group $(5.8 \% \pm 2.1 \%)$ $(P=0.03)$. In the subgroup of patients with low serum periostin levels at week 12 (C), the increase from baseline in mean ( \pm SE) FEV , was similar in the lebrikizumab group $(5.1 \% \pm 2.4 \%)$ to the placebo group $(3.5 \% \pm 2.1 \%) ;(P=0.61)$. From The New England Journal of Medicine, Corren J, Lemanske RF, Hanania NA, et al, Lebrikizumab treatment in adults with asthma. 12, 1088-1098. ${ }^{26}$ Copyright (C) 201I Massachusetts Medical Society. Reprinted with permission from Massachusetts Medical Society.

Abbreviation: $\mathrm{FEV}_{1}$, forced expiratory volume in I second.

IgE level, blood eosinophil count, and serum periostin level. The improvement in mean $\mathrm{FEV}_{1}$ was $8.2 \%$ compared with placebo $(P=0.02)$ in the high-periostin subgroup $(P=0.03)$, whereas in the low-periostin subgroup, the increase in $\mathrm{FEV}_{1}$ was $1.6 \%$ compared with placebo $(P=0.61)$ (Figure 2$)$. The fall in $\mathrm{Fe}_{\mathrm{NO}}$ was greater in the high-periostin subgroup compared to the low-periostin subgroup (34.4\% vs $4.3 \%$; $P<0.001)$. At 24 weeks, systemic biomarkers of Th2 inflammation (serum CCL13, CCL17, and total IgE levels) decreased, and peripheral blood eosinophil counts slightly increased in the group treated with lebrikizumab.

A post hoc analysis of the MILLY trial, presented at the American Thoracic Society meeting in 2012, reported that lebrikizumab treatment reduced serum periostin levels in asthma patients with elevated baseline levels of periostin, whereas low-periostin patients exhibited no significant reduction in response to lebrikizumab. ${ }^{30}$ Placebo-corrected serum periostin levels were reduced by $9.7 \%$ (95\% CI: $5.5 \%-14 \% ; P=0.001)$ at 12 weeks in the total study group. In high-periostin patients, the reduction in serum periostin was $14.4 \%$ (95\% CI: 8.3\%-20.5\%; $P<0.001)$, while in low-periostin patients, it was only $2.9 \%(95 \%$ CI: $2.5 \%-8.4 \% ; P=0.3) .{ }^{30}$ These findings suggest that in uncontrolled asthma patients, excess serum periostin is due to the activity of IL-13, and that inhibition of IL-13 with lebrikizumab decreases serum periostin levels. In summary, Phase II randomized controlled clinical trials report that lebrikizumab treatment for 12 weeks reduces late asthmatic responses after allergen challenge and improves lung function, and that this effect occurs in those subjects with a high pretreatment level of serum periostin. A dose-ranging study of lebrikizumab in adult patients who are not taking inhaled corticosteroids has been completed, but the results have not yet been reported (ClinicalTrials.gov Identifier: NCT00971035, MOLLY trial). Two large Phase III randomized controlled trials are underway that will help establish the safety and efficacy of lebrikizumab in the treatment of asthma: these trials will each aim to recruit 1400 patients with uncontrolled asthma who are receiving inhaled corticosteroids and a second controller medication. Treatment with lebrikizumab or placebo will be for 52 weeks, and the primary outcome measure is the asthma exacerbation rate (ClinicalTrials.gov Identifiers: NCT01545440, LUTE trial and NCT01545453, VERSE trial). The estimated study completion date for both trials is 2017.

\section{Other IL- I 3 blockers}

Lebrikizumab has not been compared with other therapies for asthma. A number of clinical trials employing other monoclonal antibodies targeting IL-13 in asthma have reported their findings, and further studies are underway. ${ }^{31,32}$ Tralokinumab is a human recombinant monoclonal antibody that specifically binds human IL-13 and blocks interaction with the IL-13 receptor. ${ }^{33}$ In experimental animal antigen challenge models, tralokinumab inhibited airways hyperresponsiveness and bronchoalveolar lavage eosinophilia. ${ }^{33}$ A Phase IIa study reported improvements in $\mathrm{FEV}_{1}$ and forced vital capacity (FVC) (both secondary outcome measures), but not in ACQ-6 score (primary outcome measure), with tralokinumab treatment compared with placebo, over a 12-week period, in 194 patients with moderateto-severe uncontrolled asthma despite controller therapies. ${ }^{34}$ 
A Phase IIb, 52-week randomized, double-blind study to evaluate the efficacy of tralokinumab in adults with uncontrolled, severe asthma is underway (NCT01402986). The study aims to enroll 390 participants. The primary outcome measure is exacerbations over the 52 weeks of the study. IMA-638, a humanized $\operatorname{IgG}_{1}$ antibody that neutralizes IL-13 bioactivity, reduced early and late allergen-induced responses, but with no effect on allergen-induced airway hyperresponsiveness or sputum eosinophils. ${ }^{35} \mathrm{~A}$ randomized, placebo-controlled study of 24 weeks of intravenous treatment with QAX576, a humanized IL-13-specific monoclonal antibody, has been completed in patients with persistent asthma not adequately controlled with inhaled corticosteroids and LABAs (NCT01130064). The primary outcome measure is ACQ score. To date, the results of the study have not been published.

Pitrakinra, a recombinant human IL-4 variant that is a potent inhibitor of both the IL-4 and IL-13 receptors reduced the allergen-induced late response. ${ }^{36}$ A Phase IIb doubleblind, randomized, placebo-controlled, dose-ranging study in 534 patients with moderate to severe asthma, who were inadequately controlled on the combination of inhaled corticosteroids and LABA, reported at the European Respiratory Society Meeting in 2010, found that the addition of inhaled pitrakinra failed to show clinical benefit compared with placebo. ${ }^{37}$ However, pitrakinra treatment produced a $37 \%$ reduction $(P<0.004)$ in the incidence of asthma exacerbations in a subset of patients with eosinophilic asthma $(\mathrm{n}=125)$, as measured by elevated blood eosinophils, compared to placebo. This molecule is no longer under development for the treatment of asthma.

\section{Safety and tolerability}

In a randomized controlled trial of lebrikizumab treatment administered for 24 weeks to 219 adults with asthma, musculoskeletal side effects occurred more often with lebrikizumab (13.2\%) than with placebo $(5.4 \% ; P=0.045) .{ }^{26}$ Participants were followed up for 8 weeks after the last dose of treatment. The overall frequency of adverse events was similar in the two groups $(74.5 \%$ in the lebrikizumab group and $78.6 \%$ in the placebo group), as was the frequency of severe adverse events $(3.8 \%$ in the lebrikizumab group and $5.4 \%$ in the placebo group).

Biologic agents are protein base products and as such have the potential to induce an immune response. ${ }^{38}$ The tendency to cause an antibody response to biologic agents, termed immunogenicity, is greatly reduced by the development of humanized antibodies, as is the case with lebrikizumab, but careful monitoring for adverse events will be required during the clinical development of lebrikizumab.

\section{Patient profiling and selection for lebrikizumab therapy}

In recent years, as our understanding of the different molecular and inflammatory processes has improved, there has been a move away from considering asthma as a single disease entity. Asthma is now considered a heterogenous disease consisting of several distinct asthma phenotypes. ${ }^{39}$ This approach may have clinical relevance when considering targeted asthma therapies. For example, an earlier study in patients with moderate-to-severe asthma treated with mepolizumab, a monocolonal antibody against IL-5, which is a selective and effective inhibitor of eosinophilic inflammation, failed to demonstrate any clinical benefit overall. ${ }^{40}$ However, in a subsequent study where only asthmatic patients with a refractory eosinophilic asthmatic inflammatory profile were recruited, mepolizumab resulted in a significant reduction in asthma exacerbations and improvement of quality of life ${ }^{41}$ highlighting the importance of patient profiling. Patient profiling in asthma is also performed in other standard therapies, including the anti-IgE therapy, omalizumab, ${ }^{7,8}$ and there is also evidence that titrating inhaled corticosteroid treatment to minimize sputum eosinophilia can lead to a reduction in asthma exacerbations. ${ }^{42}$

Recently, an asthma phenotype termed "IL-13 signature surrogate" has been described that is associated with a high-Th2 profile, including elevated total IgE and peripheral blood eosinophilia. ${ }^{24}$ Targeting treatment with the anti-IL-13 therapy, lebrikizumab, to those asthmatic patients demonstrating the high-Th2 status is associated with enhanced benefits, including improvement in lung function. ${ }^{26} \mathrm{~A}$ highTh2 status in an asthmatic patient is defined as a total serum IgE of more than $100 \mathrm{IU}$ per milliliter and a peripheral blood eosinophil count of $0.14 \times 10^{9}$ cells per liter or more. ${ }^{26}$

In clinical practice, surrogate markers of IL-13 activity are necessary, given that highly sensitive assays that are not clinically practical are required to quantify IL-13 in blood or airway samples. Periostin is a secreted protein originally isolated from an osteoblast cell line and is involved in cell adhesion. ${ }^{18}$ Periostin is upregulated in lung epithelial cells by IL-13 and, as such, is a potential surrogate marker of IL-13 activity; elevated levels of periostin are associated with the high-Th2 phenotype. ${ }^{20}$ Lebrikizumab treatment is associated with greater improvement in lung function in patients with elevated serum periostin levels (median value or higher) compared with those with low periostin levels 
(less than median value). ${ }^{26}$ Further prospective studies are required to confirm whether elevated serum periostin predicts response to lebrikizumab and to identify optimum cutoff points for serum periostin levels.

In a post hoc analysis, high baseline levels of $\mathrm{Fe}_{\mathrm{NO}}$, as compared with low baseline $\mathrm{Fe}_{\mathrm{NO}}$, were associated with greater efficacy of lebrikizumab in improving lung function, and higher baseline $\mathrm{Fe}_{\mathrm{NO}}$ was associated with a lower rate of severe exacerbations among patients receiving lebrikizumab than among those receiving placebo. ${ }^{26}$ However, further prospective studies are required to assess the role of $\mathrm{Fe}_{\mathrm{NO}}$ in predicting response to lebrikizumab. Induced sputum eosinophil analysis has not been assessed as a predictor of response to lebrikizumab, and this may also be worth investigating in future.

To date, lebrikizumab treatment has only been assessed in patients with poorly controlled asthma (on a 5-point asthmacontrol questionnaire, a score of 1.5 or higher), despite inhaled corticosteroid treatment of between 200-100 $\mu \mathrm{g}$ of fluticasone or equivalent per day, with prebronchodilator $\mathrm{FEV}_{1}$ between $40 \%$ and $80 \%$ of the predicted value, and with a minimum of $12 \%$ improvement after inhalation of shortacting beta agonist. Further studies may include other clinical parameters, such as exacerbation rates, that may prove helpful in identifying the optimum patient profile characteristics in predicting response to lebrikizumab.

The future treatment of asthma will see greater patient profiling and selection based on asthma phenotypes. Lebrikizumab therapy, like other recent treatments, including mepolizumab and omalizumab, would appear to support this more targeted and personalized management approach.

\section{Conclusions and future directions}

Lebrikizumab, an IL-13 inhibitor, demonstrates benefits in patients with poorly controlled asthma. The late asthmatic response after inhaled allergen challenge is reduced by almost $50 \%$ following treatment with lebrikizumab. In a Phase II study performed in 219 adults with poorly controlled asthma despite inhaled corticosteroids (MILLY trial), lebrikizumab produced an improvement in prebronchodilator $\mathrm{FEV}_{1}$ of $5.5 \%$ compared with placebo at 12 weeks, but had no effects on other efficacy end points, including asthma control and exacerbations. The benefits from lebrikizumab appear to be more pronounced in patients with a high-Th2 profile associated with elevated levels of total IgE, serum eosinophilia, and periostin. Adverse effects appear to be similar to placebo, except that musculoskeletal side effects occur slightly more often with lebrikizumab. Ongoing studies in patients with uncontrolled asthma are underway to establish the safety and efficacy of lebrikizumab when administered over a longer period. These studies will also help to determine whether identifying patients with a high-Th2 inflammatory phenotype using serum periostin as a surrogate measure permits a personalized approach to the treatment of asthma.

The studies to date have been relatively short term, and longer studies with long-term pharmacosurveillance are required to provide greater evidence of both the efficacy and safety profile of these drugs in different groups. Lebrikizumab appears to target patients with higher levels of serum periostin, which is involved in subepithelial fibrosis, raising the possibility of altering airway remodeling over the long term.

Future studies will need to address how lebrikizumab fits into the management of asthma alongside the other novel therapies. It is likely that this will require comparison of lebrikizumab with other IL-13 blockers and other biological agents such as IL-5 antagonists. These agents will need to show equal or greater benefits than more established therapies such as anti-IgE treatment. Given the heterogeneity of asthma, it may be that a combination of biological agents is required to treat the various asthmatic phenotypes.

Currently, costs are high for biological agents. Future study will need to assess the cost-benefit ratio, and will need to consider the effects on health care utilization. As such, it is likely that lebrikizumab and similar agents will be limited to those patients with more severe refractory asthma. We hope that in the future, with the advances in the phenotyping of asthma and the development of targeted treatments for these phenotypes there is the potential for greater personalized asthma therapy.

\section{Acknowledgments/disclosure}

NT has participated in advisory boards and/or received consultancy fees from Asmacure, Boston Scientific, Chiesi, Respivert. He has received lecture fees from AstraZeneca, Chiesi, GlaxoSmithKline, and Novartis and has received industry-sponsored grant funding from Aerovance, Asthmatx, AstraZeneca, Centocor, Genentech, GlaxoSmithKline, MedImmune, Novartis, and Synairgen for participating in clinical trials. MP has received lecture fees from AstraZeneca, Chiesi, and GlaxoSmithKline. AS has received lecture fees from AstraZeneca, Chiesi, GlaxoSmithKline, and Novartis.

\section{References}

1. Robinson DS, Hamid Q, Ying S, et al. Predominant Th2-like bronchoalveolar T-lymphocyte population in atopic asthma. $N$ Engl $J$ Med. 1992;326:298-304.

2. Holt PG, McCaubus C, Stumbles PA, Sly PD. The role of allergy in the development of asthma. Nature. 1999;402:B12-B17. 
3. Barnes PJ. Immunology of asthma and chronic obstructive pulmonary disease. Nat Rev Immunol. 2008;8(3):183-192.

4. Szefler S, Martin R, King T, et al. Significant variability in response to inhaled corticosteroids for persistent asthma. J Allergy Clin Immunol. 2002;109(3):410-418.

5. Bateman ED, Boushey HA, Bousquet J, et al. Can guideline-defined asthma control be achieved?: the gaining optimal asthma control study. Am J Respir Crit Care Med. 2004;170(8):836-844.

6. Anderson G. Endotyping asthma: new insights into key pathogenic mechanisms in a complex, heterogeneous disease. Lancet. 2008;372(9643):1107-1119.

7. Humbert M, Beasley R, Ayres J, et al. Benefits of omalizumab as add-on therapy in patients with severe persistent asthma who are inadequately controlled despite best available therapy (GINA 2002 step 4 treatment) INNOVATE. Allergy. 2005;60(3):309-316.

8. Thomson NC, Chaudhuri R. Omalizumab: clinical use for the management of asthma. Clin Med Insights: Circ Respir Pulm Med. 2012;6:27-40.

9. Thomson N, Chaudhuri R, Spears M. Emerging therapies for severe asthma. BMC Med. 2011;9(1):102.

10. Holgate ST. Trials and tribulations in identifying new biologic treatments for asthma. Trends in Immunol. 2012;33(5):238-246.

11. Grunig G, Warnock M, Wakil AE, et al. Requirement for IL-13 independently of IL-4 in experimental asthma. Science. 1998;282(5397):2261-2263.

12. Wills-Karp M, Luyimbazi J, Xu X, et al. Interleukin-13: central mediator of allergic asthma. Science. 1998;282:2258-2261.

13. Hershey GKK. IL-13 receptors and signaling pathways: an evolving web. J Allergy Clin Immunol. 2003;111(4):677-690.

14. Howard TD, Whittaker PA, Zaiman AL, et al. Identification and association of polymorphisms in the Interleukin-13 gene with asthma and atopy in a Dutch population. Am J Respir Cell Mol Biol. 2001;25(3):377-384.

15. Hunninghake GM, Soto-Quiros ME, Avila L, et al. Polymorphisms in IL13, total IgE, eosinophilia, and asthma exacerbations in childhood. J Allergy Clin Immunol. 2007;120(1):84-90.

16. Saha SK, Berry MA, Parker D, et al. Increased sputum and bronchial biopsy IL-13 expression in severe asthma. J Allergy Clin Immunol. 2008;121(3):685-691.

17. Barnes PJ, Adcock IM. Glucocorticoid resistance in inflammatory diseases. Lancet. 2009;373(9678):1905-1917.

18. Sidhu SS, Yuan S, Innes AL, et al. Roles of epithelial cell-derived periostin in TGF- $\beta$ activation, collagen production, and collagen gel elasticity in asthma. Proc Nat Acad Sci U S A. 2010;107(32):14170-14175.

19. Woodruff PG, Boushey HA, Dolganov GM, et al. Genome-wide profiling identifies epithelial cell genes associated with asthma and with treatment response to corticosteroids. Proc Nat Acad Sci U S A 2007;104(40):15858-15863.

20. Takayama G, Arima K, Kanaji T, et al. Periostin: a novel component of subepithelial fibrosis of bronchial asthma downstream of IL-4 and IL-13 signals. J Allergy Clin Immunol. 2006;118(1):98-104.

21. Haldar P, Pavord ID. Noneosinophilic asthma: a distinct clinical and pathologic phenotype. J Allergy Clin Immunol. 2007;119(5):1043-1052.

22. Douwes J, Gibson P, Pekkanen J, Pearce N. Non-eosinophilic asthma: importance and possible mechanisms. Thorax. 2002;57(7):643-648.

23. Wenzel SE. Asthma: defining of the persistent adult phenotypes. Lancet. 2006;368(9537):804-813.

24. Woodruff PG, Modrek B, Choy DF, et al. T-helper Type 2-driven inflammation defines major subphenotypes of asthma. Am J Respir Crit Care Med. 2009;180(5):388-395.

Biologics: Targets \& Therapy

\section{Publish your work in this journal}

Biologics: Targets \& Therapy is an international, peer-reviewed journal focusing on the patho-physiological rationale for and clinical application of Biologic agents in the management of autoimmune diseases, cancers or other pathologies where a molecular target can be identified This journal is indexed on PubMed Central, CAS, EMBase, Scopus
25. Drazen JM. A step toward personalized asthma treatment. $N$ Engl J Med. 2011;365(13):1245-1246.

26. Corren J, Lemanske RF, Hanania NA, et al. Lebrikizumab treatment in adults with asthma. N Engl J Med. 2011;365(12):1088-1098.

27. Kioi M, Seetharam S, Puri RK. N-linked glycosylation of IL-13R $\alpha 2$ is essential for optimal IL-13 inhibitory activity. FASEB J. 2006;20(13):2378-2380.

28. Scheerens H, Arron JR, Su Z, et al. Predictive and pharmacodynamic biomarkers of interleukin-13 blockade: effect of lebrikizumab on late phase asthmatic response to allergen challenge. JAllergy Clin Immunol. 2011;127(Suppl 2):AB164.

29. McClintock D, Corren J, Hanania N, et al. Lebrikizumab, an antiIL-13 monoclonal antibody, reduces severe asthma exacerbations over 32 weeks in adults with inadequately controlled asthma. Am J Respir Crit Care Med.2012;185:A3959. Proceedings of the American Thoracic Society International Conference; 2012 May 18-23; San Francisco, CA, USA.

30. Scheerens H, Arron J, Choy D, Mosesova S, Lal P, Matthews J. Lebrikizumab treatment reduces serum periostin levels in asthma patients with elevated baseline levels of periostin. Am J Respir Crit Care Med. 2012;185:A3960. Proceedings of the American Thoracic Society International Conference; 2012 May 18-23; San Francisco, CA, USA.

31. Corren J. Cytokine inhibition in severe asthma: current knowledge and future directions. Curr Opin Pulm Med. 2011;17:29-33.

32. Elias JA, Lee CG. IL-13 in asthma: the successful integration of lessons from mice and humans. Am J Respir Crit Care Med. 2011;183(8):957-958.

33. May RD, Monk PD, Cohen ES, et al. Preclinical development of CAT-354, an IL-13 neutralizing antibody, for the treatment of severe uncontrolled asthma. Br J Pharmacol. 2012;166(1):177-193.

34. Piper E, Brightling C, Niven R, et al. A phase 2 placebo-controlled study of tralokinumab in moderate-to-severe asthma. Eur Respir J. Epub June 27, 2012.

35. Gauvreau GM, Boulet L-P, Cockcroft DW, et al. Effects of interleukin-13 blockade on allergen-induced airway responses in mild atopic asthma. Am J Respir Crit Care Med. 2011;183(8):1007-1014.

36. Wenzel S, Wilbraham D, Fuller R, Getz EB, Longphre M. Effect of an interleukin-4 variant on late phase asthmatic response to allergen challenge in asthmatic patients: results of two phase 2a studies. Lancet. 2007;370(9596):1422-1431.

37. Wenzel S, Ind P, Otulana B, Bleecker E, Kuna P, Yen Y. Inhaled pitrakinra, an IL-4/IL-13 antagonist, reduced exacerbations in patients with eosinophilic asthma. Proceedings of the European Respiratory Society Annual Congress; 2010 Sept 18-20; Barcelona, Spain.

38. Purcell R, Lockey R. Immunologic responses to therapeutic biologic agents. J Investig Allergol Clin Immunol. 2008;18(5):335-342.

39. Wenzel SE. Asthma phenotypes: the evolution from clinical to molecular approaches. Nat Med. 2012;18(5):716-725.

40. Flood-Page P, Swenson C, Faiferman I, et al. A study to evaluate safety and efficacy of mepolizumab in patients with moderate persistent asthma. Am J Respir Crit Care Med. 2007;176(11):1062-1071.

41. Haldar P, Brightling CE, Hargadon B, et al. Mepolizumab and exacerbations of refractory eosinophilic asthma. $N$ Engl J Med. 2009;360(10):973-984.

42. Green RH, Brightling CE, McKenna S, et al. Asthma exacerbations and sputum eosinophil counts: a randomised controlled trial. Lancet. 2002;360(9347):1715-1721.

and the Elsevier Bibliographic databases. The manuscript management system is completely online and includes a very quick and fair peerreview system, which is all easy to use. Visit http://www.dovepress. $\mathrm{com} /$ testimonials.php to read real quotes from published authors. 